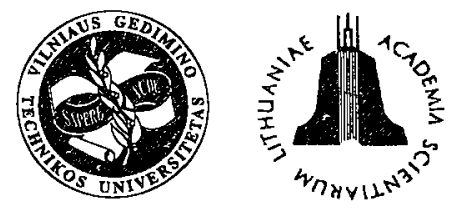

\title{
DYNAMIC PROCESSES IN THE MIAIN OIL PIPELINES. PART I. THE ANALYSIS OF CAUSES OF LEAKAGE OCCURRENCE IN OIL PIPELINES AND LEAK IDENTIFI- CATION METHODS
}

\author{
Ingrida Bajoraityṫ̇ ${ }^{1}$, Marijonas Bogdevičius ${ }^{2}$ \\ Department of Transport Technological Equipment, Vilnius Gediminas Technical University, \\ Plytines g. 27, LT-2016 Vilnius, Lithuania, Tel. (5) 699-705
}

Received 200206 26; accepted 20021021

\begin{abstract}
The causes of accidental damages and the damages in oil pipelines are presented. The analysis of identification methods of leaks occurring due to the damages of the linear part of the main oil pipelines is carried out. The momentum and continuity equations of viscous compressible fluid in a pressure pipeline are presented. Differential equations of fluid movement in the oil pipeline are solved by the method of characteristics. Variation diagrams of pressure and velocity of fluid of a leaking oil pipeline are presented.
\end{abstract}

Keywords: oil pipeline, damage, identification, numerical method, wave speed.

\section{Introduction}

Since the development of various industrial branches requires more and more power resources, modern main oil pipelines have been exploited intensively. To raise the efficiency of the main oil pipelines, pressure must be increased. Working at the maximum permitted pressure the probability of accidents in the main pipelines increases sharply. To identify leaks immediately, automatized systems should be implemented.

Such systems are indispensable for the identification of unauthorized connections to the main pipelines as well.

Since the proportion of the fluid transported through these connections is rather small, the identification of leaks is very complicated.

\section{Causes of Accidental Damages in the Main Pipe- lines}

The causes of accidental damages in the main pipelines can vary a lot. Accidental damages of pipelines can occur due to physical and chemical impacts. Physical impact may be caused by external and internal forces influencing the pipeline which cause mechanical tensions or fatigue phenomena in the material of a pipe. External mechanical impacts might be caused for example, by ground water which washes off the supports of a pipeline, falling ground, settling or sliding land as well as seismological vibrations.

Internal forces can form in a pipeline due to exceeded

\footnotetext{
${ }^{1}$ E-mail: inga@post.omnitel.net

${ }^{2}$ E-mail: marius@ti.vtu.lt
}

pressure, which might be caused by the rapid throttling of a pipeline transmitting cross-section, starting or stopping machines (hydraulic impact), resonance phenomena of the primed product pressure pulsation, etc. as well as fluently increasing the pressure over the permitted limit, e.g. gradually decreasing the fluid yield in a pipeline. The strength limit of pipeline material, pipes and their joints varies with the exception of welded joints which are not weaker than the material of a pipeline. Statistics shows that $20 \%$ of pipeline damages occur due to invisible defects (micro cracks, depressions, etc.) occurring in pipelines prior to laying them in the ground. Defects occur when pipes are manufactured in a plant or mounted and are not noticed during testing. Due to internal and external forces such defects gradually increase and can cause damages or even major cracks in pipelines which cause leakage of a transported product.

Chemical impact is chemical or electrochemical corrosion of a pipeline material. Minor leakage normally occurs due to the corrosion of the wall of a pipe. Due to corrosion the walls of a pipeline gradually become thinner; therefore mechanical tensions increase in the metal of a pipeline. When mechanical tensions in the wall of a pipeline exceed the limit of strength, a pipeline breaks. Physical impact is usually short-term: from one second to several hours or 24 hours; whereas chemical impact can last for months and even years. During exploitation minor damages of a pipe tightness cause leakage of a transported product as well as major accidental damages. Expansion speed of a crack in a steel pipe is $\approx$ $1220 \mathrm{~m} / \mathrm{s}$ and the duration of the cracking process is calculated per one thousandth of a second, i.e., crack occurs instantly [1]. 


\section{The processes of an accident are divided into two} stages:

1) rapid spraying of oil through an open crack;

2) increase of a crack and a slower abundant flow of oil to a complete fall of pressure by closing valves.

Various factors cause circular and longitudinal tensions as well as tensions caused by bending eflections which cause longitudinal and transverse displacement. During exploitation longitudinal tensions occur as well which are mostly noticed at the bends of a line as well as near valves.

Longitudinal tensions will always be less than transverse; therefore, a pipe usually breaks transversely to the pipe axle.

The ground layer above a pipe causes normal pressure which influences the walls of a pipe and resists to longitudinal and transverse displacement of a pipeline occurring due to pressure and temperature.

Following a long-term practice of the main pipelines exploitation it is impossible to ensure their proper condition due to external factors as well as due to other reasons. Therefore accidental damages occur in the main oil pipelines.

Damages of pipes, cracks and leakage of the main oil pipelines are influenced by the following factors:

- Manufactural defects of pipes: metallurgical defects (peeling of pipe walls, non-metal alloys); exploitation of non-calculated resistance, plasticity and fluidity characteristics; deviations of geometrical characteristics from the project (thickness of a pipe wall and diameter of a pipe); defects of manufactural welding of joints (bad quality welding when cracking is not eliminated, alloys of slacks in the joint, weakening of the main metal at the welding joint; defects of pipe manufacturing (micro cracks, scratches, cuts occurring in the metal of a pipe during the process of manufacturing).

- Defects of welding pipes when welding is carried out on the site: similar defects like during the manufacturing process in a plant (bad quality welding, unevenness of welding joint metal tensions compared to the tensions of the main metal as well as flaking of the main metal on the area around the welding joint) occur.

- $\quad$ Pipe corrosion causes formation of depressions and holes of various sizes as well decreases the thickness of a pipe wall. Several cases of corrosive damages are presented in Fig 1 a.

Continuous even corrosion covering large areas of a pipe wall, continuous uneven corrosion at the same time damaging holes of various depths at different spots on the walls of a pipe are presented. In local corrosion metal deteriorates in local areas, narrow and deep gnaws as well as complete corrosion of a wall occur.

Occurrence of negative tensions which are caused by non-compliance with the project requirements and er- rors of project solutions is a very frequent cause of damages occurring in pipelines: occurrence of tensions due to loadings not taken into consideration during the process of design. Such loadings include loading of landslides,

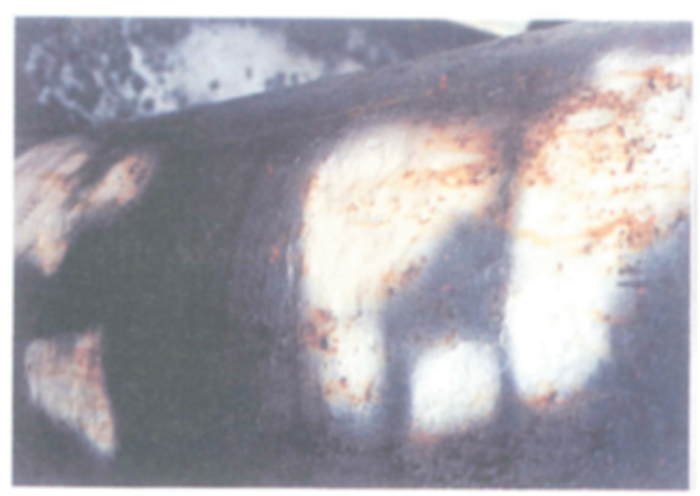

a)

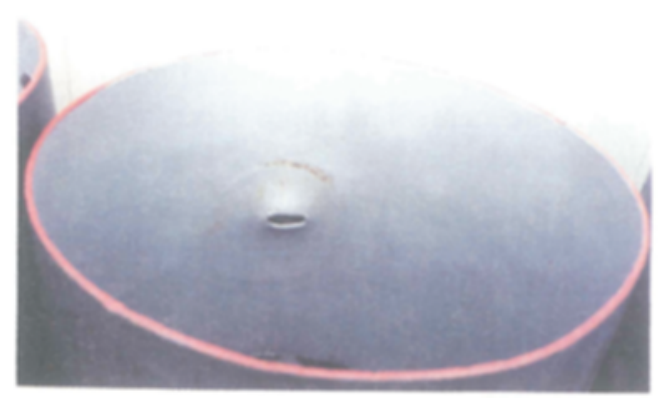

b)

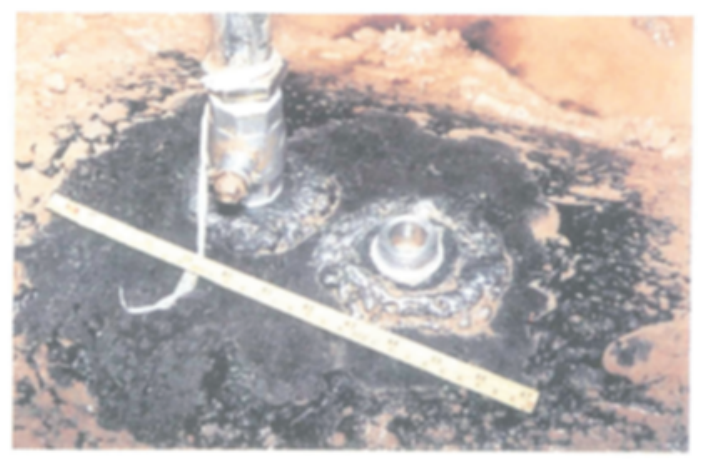

c)

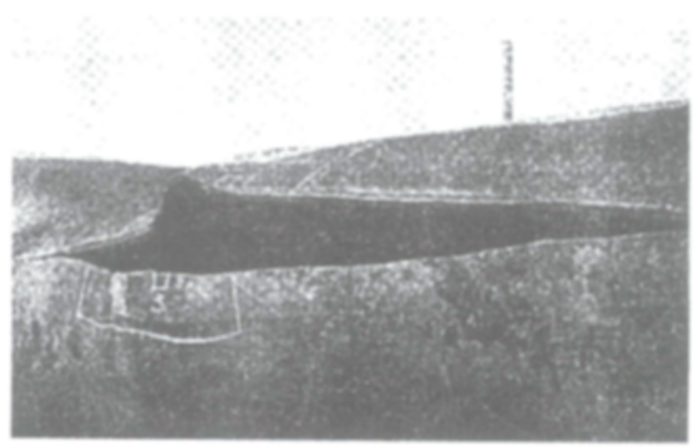

d)

Fig 1. Defects of pipes: a - corrosion covering large area of a pipeline wall; $b$ - the pipeline is punctured; c - unauthorized connection to main pipelines; $\mathrm{d}-$ along welding connection a weld 
wash-out of soil by ground waters or during heavy rain under a pipeline (occurrence of depression).

- Non-compliance with the exploitation regime occurs when exploitation pressure is exceeded, inspection of pipelines is not carried out, their condition is not assessed and dangerous areas are not identified on time (swelling, intensive corrosion, etc.) $[2,3]$.

- Mechanical damages of pipes during their transportation, laying, or exploitation are as follows: bends, scratches, over bends, tears, thinner edges of a pipe when towing them with a towing vehicle, formation of micro cracks, damages of pipelines by earth work machinery when carrying out various agricultural work.

- Illegal connection.

In most cases damages of pipelines through which oil and oil products are transported expand from tens of metres to tens of kilometres. Damages of tens of metres occur rather rarely.

Following the statistical data [4] tendencies of the variation of the number of major and minor accidents occurring in the main oil pipelines depending on the year of exploitation can be observed (see Fig 2).

Here we can see that during the first years of exploitation the number of major and minor accidents is the greatest. This is the period of the started and to be continued work of the main pipeline. During this period a majority of manufacturing defects as well as defects occurring during the laying of a pipeline and welding joints are detected. A number of minor and major accidents decreases when the period of exploitation increases. It can be noticed that the least number of accidents occurred in the seventh year of exploitation. The second and the seventh year of exploitation is a period of operational exploitation. During the eighth year of exploitation the number of major and minor accidents starts increasing again. Essential repair work must be carried out when the tendency of the increasing number of accidents is observed.

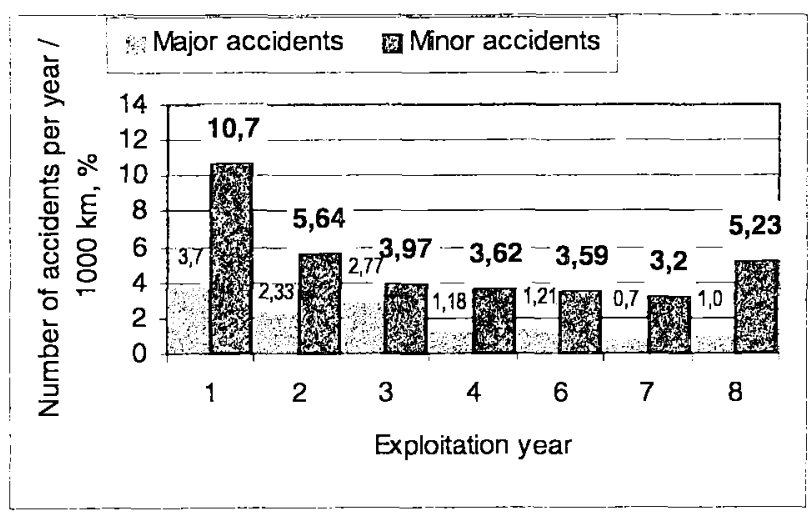

Fig 2. The tendency of the variation of the number of major and minor accidents forming in the main oil pipelines depending on the year of exploitation
3. Survey of Identification Methods of Leaks Occurring due to Damages of the Linear Part of the Main Oil Pipelines

When controlling the work regime of the main pipelines timely identification of the main oil pipeline damages and leaks is very important. The scope and impact on the environment of an accident of the linear part depend on the size of a leak. Leaks are usually classified into:

- $\quad$ minor (not exceeding $3-5 \%$ of nominal yield);

- average (up to $10 \%$ of nominal yield);

- major (exceeding $10 \%$ of nominal yield).

At present a lot of various methods to register the size, time, and location of a leak have been developed. Classification of a leak size and location identification methods can be classified into two groups:

passive (according to the data on the observation of the transported product): mixed methods; noise methods; impact waves analysis methods; pressure variation comparison method; differential-acoustic method; fluid capacity measuring and comparison method; balance method;

active (transportation of diagnosing equipment through a pipeline): External methods: method of radioactive elements usage; visual method; investigation of surrounding air (gas); acoustic noise control; Internal methods: method of using probes transported by fluid flow in a pipeline; cable usage method.

Most frequently the following combinations of methods are applied to identify the location of a leak [5].

\subsection{Passive Methods}

Method of linear balance. Following the method of linear balance, a leak in a pipeline shall be identified by graphical or calculation method according to hydraulic deviations at the beginning and at the end of the damaged pipeline. In case of leakage hydraulic deviation will be greater at the beginning of a pipeline and smaller at the end. The accuracy of this method is influenced by various physical-chemical properties of oil products as well as capacity fluctuations, temperature, pressure in pipeline and other factors. The accuracy of this method depends on the parameters of pipeline regime as well. The method of pipeline linear balance enables to identify the leakage in the limit of $1-5 \%$ flow; however, the leak cannot be identified by this method. The disadvantage of this method is time duration (up to 24 hours) required for the equalization of the flow up to exploitation regime fluctuations. Therefore, the time of reaction of the linear balance system till the occurred leakage is rather long.

Control of pressure reduction in a pipeline. The speed of pressure fall depends on the amount of leakage and the distance from the location of a damage of a pipeline. A leak can be identified by the fall of pressure in a pipeline. At present mechanical and electrical devices registering 
and signaling about the fall of pressure below the limit at a certain point of a pipeline are used. Their sensitivity is limited by pressure fluctuations in a pipeline, which are caused by exploitation factors. Moreover, to identify the location of a damage, the values of pipeline route pressure shall be known. Such systems are usually used to identify major leakage, but they do not guarantee high accuracy of identifying the location and size of leakage [5].

Method of fluid capacity measurement and comparison on pipeline sections. Capacity measuring devices are set up longitudinally at certain distances in a pipeline and their accuracy class is $0.2-0.5 \%$. The indicators are transferred via telemetric system. In the case of leakage the leak which will be in the pipeline section between two adjacent capacity measuring devices can be easily identified according to the indicators of capacity measuring devices. Following this principle it was suggested to set up differential protection of a pipeline. The disadvantages of differential protection are as follows: great amount of capacity measuring devices, special electrical communication channels necessary for connecting capacity measuring devices and supply electrical current as well as transfer signals to the switchboard operator; inaccurate identification of the leak (only on the protected section), possible incorrect signals of relay during transitional processes. These disadvantages do not allow to use the method of differential protection in pipelines.

Control of rapid variation of pressure or flow velocity in a pipeline. Following observations rapidly occurring leakage causes rapid variation of pressure on both sides of the leakage in a pipeline. Pressure can be registered with mechanical or electronical devices mounted on the ends of a pipeline section which change the speed of pressure fall. Having achieved the determined limited pressure level, emergency signaling starts. This system differs from others by its fast reaction since pressure waves in a pipeline are transferred at a high acoustic speed (ca $1000 \mathrm{~m} / \mathrm{s}$ in fluids). Sensitivity of this system when used in practise is low since relatively high pressure threshold is set to reject frequent false signals which are caused by pulling up and starting equipment being repumped as well as the work of regulating valves, etc. [6].

Noise level measuring level method. There are methods among a variety of acoustic methods based on the comparison of noise level at various spots of a pipeline. Telematic information transfer is set up for this method. The system of measuring elements is evenly distributed in the pipeline under control and connected via transfer channels with control points. Direct indication of damaged section following the method of analysis is received from the noise information of separate measuring elements which are fixed on the control point circuits by giving the sound signal. Such system is intricate and expensive. It is used when expensive products are transported via pipelines for comparatively short distances.

This method is relatively complicated. Special com- munication channels, equipment which analyzes noise composition and level, telemechanical equipment, etc. are required for this method; therefore, it is not purposeful to use them in the main pipelines since a leak can be identified with a great number of measuring elements [7].

Veifalert system. Veifalert system of directed impact is based on the registration principle of short-term pressure variations in the case of leakage in a pipeline. Dynamic measuring elements set up at the ends of the pipeline section register pressure variation waves which form on the section between stations i.e., on the protected section. The protection effect is achieved at the ends of the section when sensitive measuring elements are set up having processed special signals received from them. The system reacts to the occurrence of leakage instantenously and guarantees its localization since the system operation time is identified via transit of acoustic signal longitudinally to te pipeline line. The sensitivity of the system is good [5].

Differential-acoustic method. In the case of a pipeline damage occurring due to the leakage flow periodical, continuous fluctuations causing acoustic noise outside and inside the pipeline occurs. Due to multiple reflections to pipe couplings and energy return supports as well as ground stops longitudinally to a pipeline do not expand far. Low frequency fluctuations occurring in fluid propagate at a much larger distance. Following the law of acoustic fluctuations in a pipeline, the location of fluctuations, ie. the location of a damage can be identified measuring the noise level at the beginning and the end of a damaged section in a relatively narrow spectrum of frequencies [8].

Method of impact waves. N. Žukovskij suggested to identify locations of damages in a pipeline by an impact diagram written during the hydraulic impact which is caused rapidly closing the barrier at the end of a pipeline section. The diagram shows the variation of pressure at the barrier (at the end of a pipeline section). If the propagation speed of an impact wave is known, the location of damage in a pipeline can be identified rather accurately.

The disadvantage of N. Žukovskij method is that an impact wave propagates rapidly along the whole crosssection of a pipeline when a barrier is closed, which causes a rapid increase of pressure (hydraulic impact). It can cause additional damages of a pipeline. Modern equipment enables to measure and register small variations of flow velocity or pressure. Therefore, hydraulic impact shall not be created closing the whole cross-section of a pipeline completely. Weak hydraulic impact can be created by rapid opening or closing of a valve (e.g. with electromagnetic transmission) at a pipeline branch-off. Experiments showed that hydraulic micro impact caused under this method allows to register the distribution of variations and their reflection from the roughness of a pipeline and between them as well as reflections from the locations of damages. Following the method of N. Žukovskij, leaks in a short pipeline section can be identified by hydraulic 
micro impact: on the section of several hundred metres at minor leakage and on the section of several kilometers at great leakage.

It is recommended to apply $\mathrm{N}$. Žukovskij method identifying the leak precisely on the $1-2 \mathrm{~km}$ long pipeline section. This method is frequently used to specify the damage in the main pipeline which was identified following another method.

\subsection{Active External Methods}

Visual observation. To identify visible changes, e.g. appearance of lubricant spots, changes of plants as well as other leakage symptoms external visual method of identifying leakage is carried out walking, driving or flying (by plane or helicopter) around the pipeline. The accuracy of this method is very low and the time of identifying the location of an accident is limited by the number of inspections which cannot increase high costs of control operations. Moreover, mistakes can be made locating a leakage since a product can flow at a great distance from the location of a damaged pipeline.

Investigation of surrounding air (gas). Various devices not far from the pipeline can be used to identify the leak which react to the increased concentration of carbohydrates in the air around the leakage. These devices are usually portable. The sensitivity of devices is relatively high; however, the identification time of a leakage is limited by the frequency (number) of inspections. Moreover, an approximate size of leakage is identified.

Measuring acoustic noise. At present a lot of portable devices and equipment are used for the acoustic control of the linear part of a pipeline. The sensitivity of such devices is usually good. However, it is always required that their sensing elements contacted directly with a wall of a pipeline; whereas certain difficulties are faced examining underground or underwater lines.

Method of using radioactive elements. This method is based on the radioactive radiation. Radioactive isotopes are mixed with the product in a pipeline which creates a radioactive background in the pipeline and its concentration in the damaged section of a pipeline is extremely high.

Damages are identified with sensing elements, e.g. Geiger-Muller counter (tube). This method allows to identify damages in pipelines under a layer of soil [6].

\subsection{Active Internal Methods}

Method of using the probe transported by a fluid flow. To identify the location of damage in a pipeline probes which move together with fluid longitudinally or in separate pipeline sections are used. Various devices are mounted inside the probe which measure and register pressure or other parameters inside a pipeline. Meanwhile, the distance covered by the probe in a pipeline is fixed on a diagram.

In the case of registering pressure the location of damage can be identified by the rapid pressure fall in the area of leakage. When registering acoustic noise leakage is identified by the rapidly increased noise. The location of leakage can be identified mounting an extremely sensitive device in a probe which registers the variation of pressure.

However, probes cannot be mounted in all pipelines. Probes are used for periodical inspection of pipelines to identify the damages which cannot be identified by passive methods.

Method of using a cable. A lot of practical systems have been developed when a cable with a special coverisolation is used to diagnose leakage. Protection properties of isolation are damaged when impacted by a primed product and the leak can be identified locating a cable damage.

The reaction time of systems where various probes are used is good; however, their price is high.

Acoustic methods of leakage identification and Veifalert system methods of simulating non-stationary processes in pipelines are very efficient. All of them are based on processing information using specialized processes or serial electronical-calculated complexes. The usage of specialized calculated devices ensures high efficiency [6].

\section{Dynamic Processes in the Oil Pipeline}

The flow of viscous and compressive fluid is described by differential equations with partial derivatives which express the laws of mass movement, quantity and conservation of energy. The equation of fluid condition is added to differential equations which relate thermodynamic variables. These equations with initial and boundary conditions influence on the solution and can be essentially integrated during time. Therefore, fluid flow parameters at certain moments can be estimated.

Solving complicated applied hydrodynamic tasks non-stationary quasi-linear one-dimensional fluid flow models are widely applied [9-15]. In such models the condition of flow at every moment of time is characterized by average values of pressure, velocity and density. When estimating the average values of fluid flow parameters, the following pre-conditions are taken into consideration: pressure, density, internal energy and temperature change inconsiderably in a cross-section of a pipeline; the speed of movement on the walls of a pipeline is equal to zero.

We will study one-dimensional non-stationary isothermic movement of viscous and compressive fluid in a pipeline; i.e. when the fluid velocity vector is directed longitudinally to the axis of a pipeline and the speed and pressure change during time and longitudinally to the axis of a pipeline (coordinate $x$ ).

Momentum and continuity equations of viscous, compressible fluid in pressure pipe have the following form: 
$\frac{1}{\rho} \frac{\partial p}{\partial x}+\frac{\partial v}{\partial t}+v \frac{\partial v}{\partial x}+\frac{f v|v|}{2 d}+a_{x}=0$

(1)

$$
\frac{\partial p}{\partial t}+v \frac{\partial p}{\partial x}+c^{2} \rho \frac{\partial v}{\partial x}=0
$$

where $p, v, \rho$ - pressure, velocity and density of fluid; $c-$ wave speed; $d$ - internal diameter of a pipeline; $f$ coefficient of pressure losses along pipeline $f=f(R e)$, $a_{x}$-acceleration by $x$ direction.

Differential equations of liquid movement in the cylinder are solved by a characteristics method $[9,11]$. The main idea of characteristics method is the fact that unknown variable speed and liquid pressure at instant moment of time $t+\Delta t$ is determined according to these parameters at a moment of time (Fig. 3).

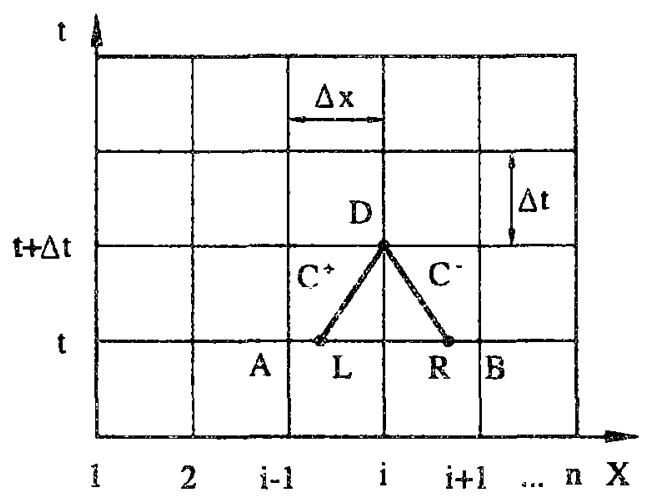

Fig 3. Scheme of point parameters determination by a characteristic method

Pressure and velocity at point $D$ at the moment of time are determined from nonlinear algebraic equation system

The velocity of sound in a two-phase liquid is placed in a pipeline as determined:

$$
c=\sqrt{\frac{K(p) / \rho}{1+\frac{K(p) \cdot d}{E \cdot \ell}+\frac{\varepsilon}{\gamma}\left[\frac{K(p)}{\gamma p}-1\right]},}
$$

where $K_{p}$-bulk modulus elasticity of fluid; $E$-modulus elasticity of pipeline;

$e$-wall thickness of the pipeline;

$\gamma$ - isentropic exponent;

$\varepsilon$ - relative volume of gas in the liquid.

Leakage in the oil pipeline is simulated closing and opening leaking pipeline. The length of the pipeline under investigation is one kilometer, the inner diameter of pipeline is equal to $0.730 \mathrm{~m}$. The density of fluid $\rho=890 \mathrm{~kg} / \mathrm{m}^{3}$, wave speed is equal to $1095 \mathrm{~m} / \mathrm{s}$. Variations of velocity and pressure in the system were observed when the diameter of the first pipe is $50 \mathrm{~mm}$ and the opening time of simulated leakage is $t_{\text {open }}=20 \mathrm{~s}$ and closing time simulating the end of leakage is $t_{\text {close }}=30 \mathrm{~s}$. The diameter of the second pipe is $75 \mathrm{~mm}$ and the opening time simulating leakage is $t_{p e n}=40 \mathrm{~s}$ and the closing time simulating the end of leakage is $t_{\text {close }}=50 \mathrm{~s}$. Diagrams of pressure and velocity variation at different diameters of leaking pipeline are presented in Fig 4.

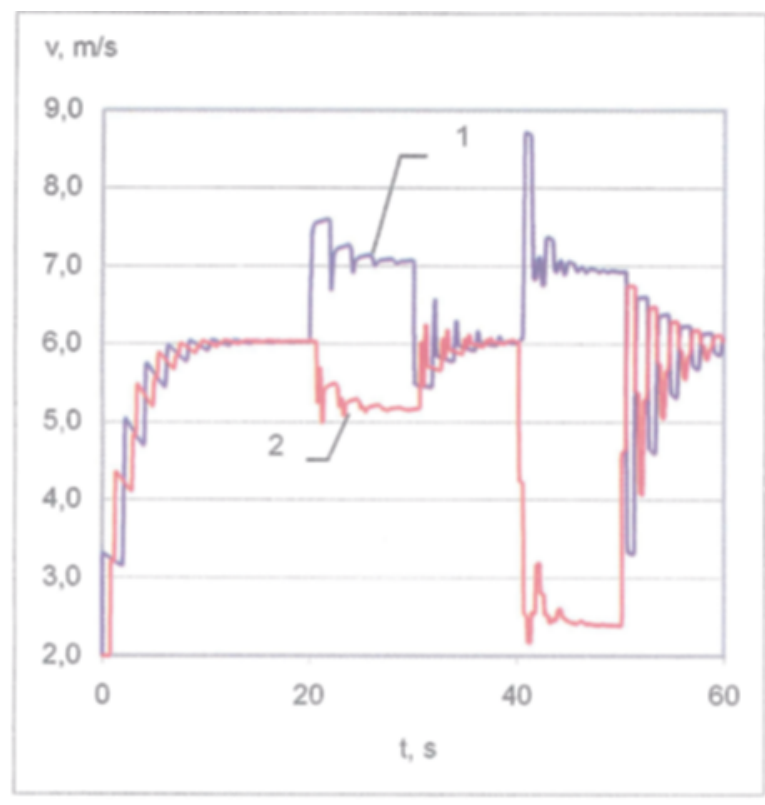

a)

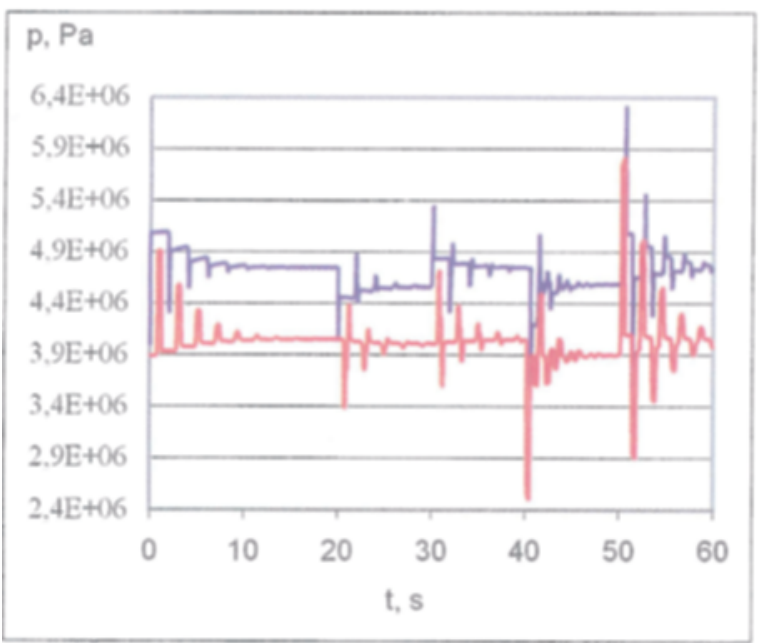

b)

Fig 4. Diagrams of parameters of fluid when the diameter of the first leaking pipeline is $50 \mathrm{~mm}$ and the diameter of the second leaking pipeline is $75 \mathrm{~mm}$; 1 - the first sensing element; 2 - the second sensing element; a - velocity; $\mathrm{b}$ - pressure

\section{Conclusions}

1. The most dangerous impact which reduces reliability and durability of the main oil pipelines is corrosion.

2. The most effective method of leak identification in the main oil pipelines is the method of impact waves. 
The method of characteristics is an effective numerical method for the research of dynamic processes in the main oil pipelines. Based on that an effective system of leak identification in the main oil pipelines can be developed.

\section{References}

1. Agapkin B. The Final Analysis of Science and Engineering. A Pipeline Transport. Moscow (Итоги науки и техники. Трубопроводный транспорт). Moscow, 1984. $105 \mathrm{p}$ (in Russian).

2. Borodavkin P. Underground Main Pipelines (Подземные магистральные трубопроводы). Moscow, 1982. 384 p (in Russian).

3. Borisov V. Control of the Main Pipelines (Управление магистральными трубопроводами). Moscow, 1979. 215 $\mathrm{p}$ (in Russian).

4. Below N. The Analysis of Accident Rates on Gas and Oil Pipelines (Анализ аварийности на газо-нефтепроводах). Moscow, 1990. 319 p (in Russian).

5. Alijev R. Pipeline Transport of Oil and Gas (Tpy6oпроводный транспорт нефти и газа.) Moscow, 1988. 368 p (in Russian).

6. Pipeline Transport. The Final Analysis of Science and Engineering, Volume 3 (Трубопроводный транспорт. Итоги науки и техники, том 3). Moscow, 1990. 120 p (in Russian).

7. Non-destructive Inspection and Diagnostics of Pipelines (Неразрушающий контроль и диагностика трубопроводов). Moscow, 1988. 159 p (in Russian).
8. Perfecting of Acoustic and Emission Search of Leakages in Pipelines. Oil and Gas Industry (Совершенствование акустоэмиссионного поиска утечек в трубопроводах). Moscow, 1986, No 2, p 51 -52 (in Russian).

9. Bogdevičius $M$. Non-stationary movement of fluid in the elastic, visco-elastic, and plastic pipe (Nestacionaraus skysčio tekèjimas tampriais, klampiais ir plastiškais vamzdžiais). Applied Mechanics (Taikomoji mechanika). 1994, No 2, p 117-124 (in Lithuanian).

10. Michael J. Here are the factors that govern evaluation of mechanical damage to pipelines. Oil \& Gas Journal, Sept. 9, 2002, p 64-69.

11. Aladjev V., Bogdevičius M. Solution of Physical, Technical and Mathematical Problems with Maple V (Решение физико-технических и математических задач с пакетом Maple V). Vilnius: Technika, 1999. 686 p (in Russian).

12. Aladjev V.; Bogdevičius M. Maple 6: Solution of the Mathematical, Statistical and Engineering-physical Problems (Maple 6: Решение математических, статистических и инженерно-физических задач). Moscow, 2001. 824 p (in Russian).

13. Aladjev V.; Bogdevičius M.; Prentkovskis O. New software for Mathematical Package Maple of Releases 6,7 and 8. Monograph. Vilnius: Technika, 2002. 404 p.

14. Bogdevičius M. Simulation of Hydraulic Systems of the Pump by the Method of Characteristics (Моделирование гидравлической системы насоса методом характеристик). Transport Engineering (Transportas), No 2, Vilnius: Technika, 1997, p 30-37 (in Russian).

15. Bogdevičius M. Simulation Interaction of Mechanical and Hydraulic System. In: Tenth World Congress on the Theory of Machines and Mechanisms. Oulu, Finland, 1999, p $2110-$ 2115. 